\title{
Chapter 9. HZPC Americas Corporation Early-Line Variety Trial, 20061
}

\author{
C.M. Hutchinson, and Doug Gergela ${ }^{2}$
}

\begin{tabular}{|c|c|}
\hline \multicolumn{2}{|c|}{$\begin{array}{l}\text { A goal of this trial was to evaluate the production characteristics of early generation clones and advanced } \\
\text { varieties from HZPC Americas Corporation in Florida. Many of these European lines have unique quality } \\
\text { characteristics that could broaden the definition of "new" Florida potato beyond the standard Red LaSoda! } \\
\text { Planting Information }\end{array}$} \\
\hline Planting Site & PSREU - Hastings Farm, Hastings, FL \\
\hline Planting Date & February 1, 2006 \\
\hline Vine Kill Date & April 28, 2006 \\
\hline Harvest Date & May 23 and 24,2006 \\
\hline Season Length & 100 days planting to vine kill; 112 days planting to harvest \\
\hline Fertilizer Program & preplant, 100-43-86 lb/A; sidedress, 65-0-56 lb/A (2 appl.) \\
\hline Irrigation Program & seepage \\
\hline \multicolumn{2}{|l|}{ Experimental Design } \\
\hline Number of Varieties & 13 \\
\hline Number of Clones & 221 \\
\hline Seed Tuber Type & cut seed \\
\hline Within Row Spacing & $8 \mathrm{in}(20 \mathrm{~cm})$ \\
\hline Between Row Spacing & 40 in $(102 \mathrm{~cm})$ \\
\hline Replications & 1 replication observational trial \\
\hline Plot Size & $10.7 \mathrm{ft}(3.3 \mathrm{~m})$ \\
\hline \multicolumn{2}{|l|}{ Production Statistics } \\
\hline Early Vigor Ratings & 41 days after planting \\
\hline Highest Total Yield & HZPC 43 (602 cwt/A or $67.4 \mathrm{MT} / \mathrm{ha})$ \\
\hline Highest Marketable Yield & HZPC 11 (438 cwt/A or $49.1 \mathrm{MT} / \mathrm{ha})$ \\
\hline Best Appearance Rating & HZPC 76,78 , and $80(7.5)$ \\
\hline
\end{tabular}

1. This document is HS1093, one of a series of the Horticultural Sciences Department, Florida Cooperative Extension Service, Institute of Food and Agricultural Sciences, University of Florida. Original publication date May, 2007. Visit the EDIS Web Site at http://edis.ifas.ufl.edu.

2. Chad M. Hutchinson, Associate Professor, Doug gergela, Sr. Biological Scientist, Horticultural Sciences Department, Cooperative Extension Service, Institute of Food and Agricultural Sciences, University of Florida, Gainesville, 32611

The use of trade names in this publication is solely for the purpose of providing specific information. UF/IFAS does not guarantee or warranty the products named, and references to them in this publication does not signify our approval to the exclusion of other products of suitable composition. All chemicals should be used in accordance with directions on the manufacturer's label. Use pesticides safely. Read and follow directions on the manufacturer's label.

The Institute of Food and Agricultural Sciences (IFAS) is an Equal Opportunity Institution authorized to provide research, educational information and other services only to individuals and institutions that function with non-discrimination with respect to race, creed, color, religion, age, disability, sex, sexual orientation, marital status, national origin, political opinions or affiliations. U.S. Department of Agriculture, Cooperative Extension Service, University of Florida, IFAS, Florida A. \& M. University Cooperative Extension Program, and Boards of County Commissioners Cooperating. Larry Arrington, Dean 


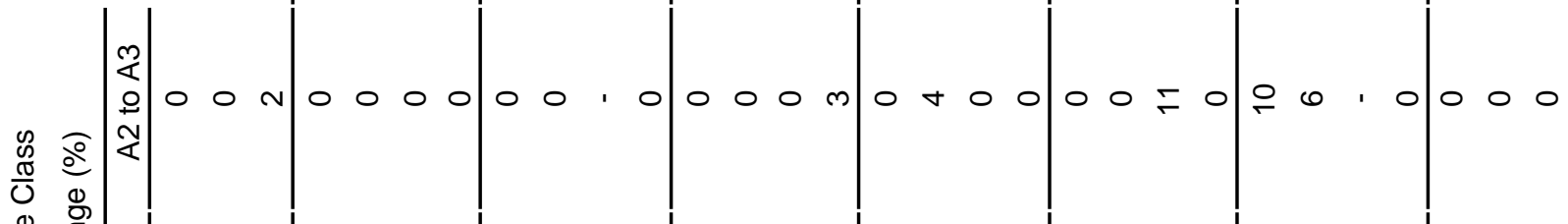

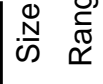

悉

至

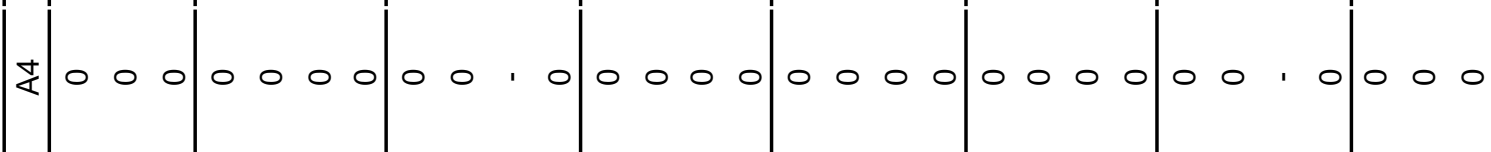

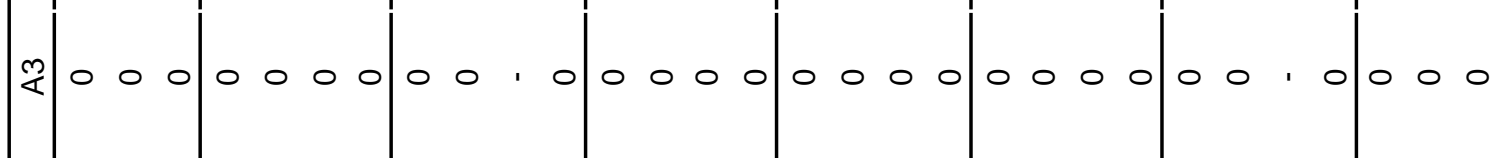

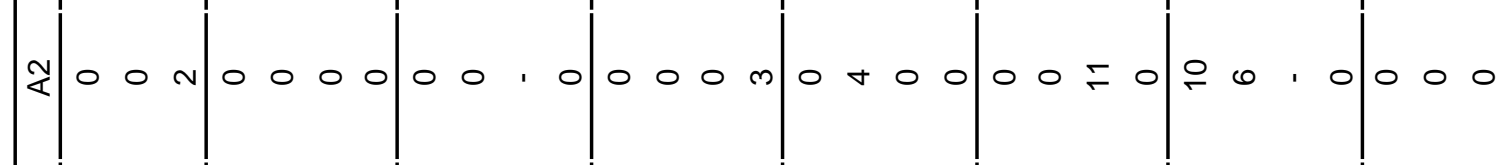

幽。

๓

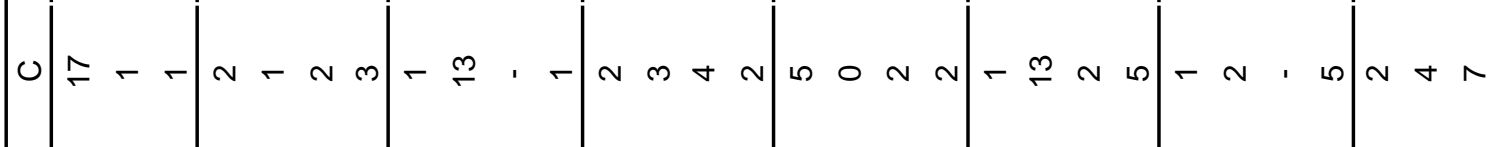

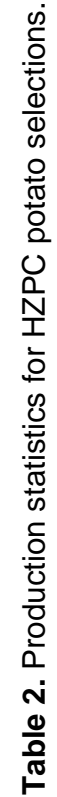

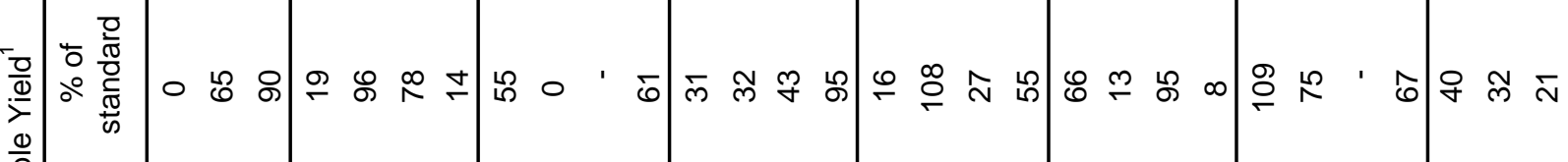

疍

恶

ำ

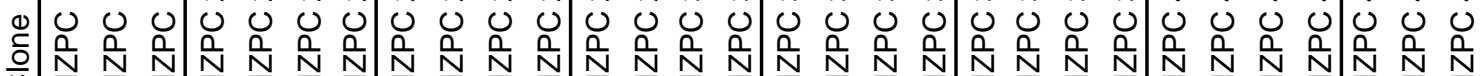
o 


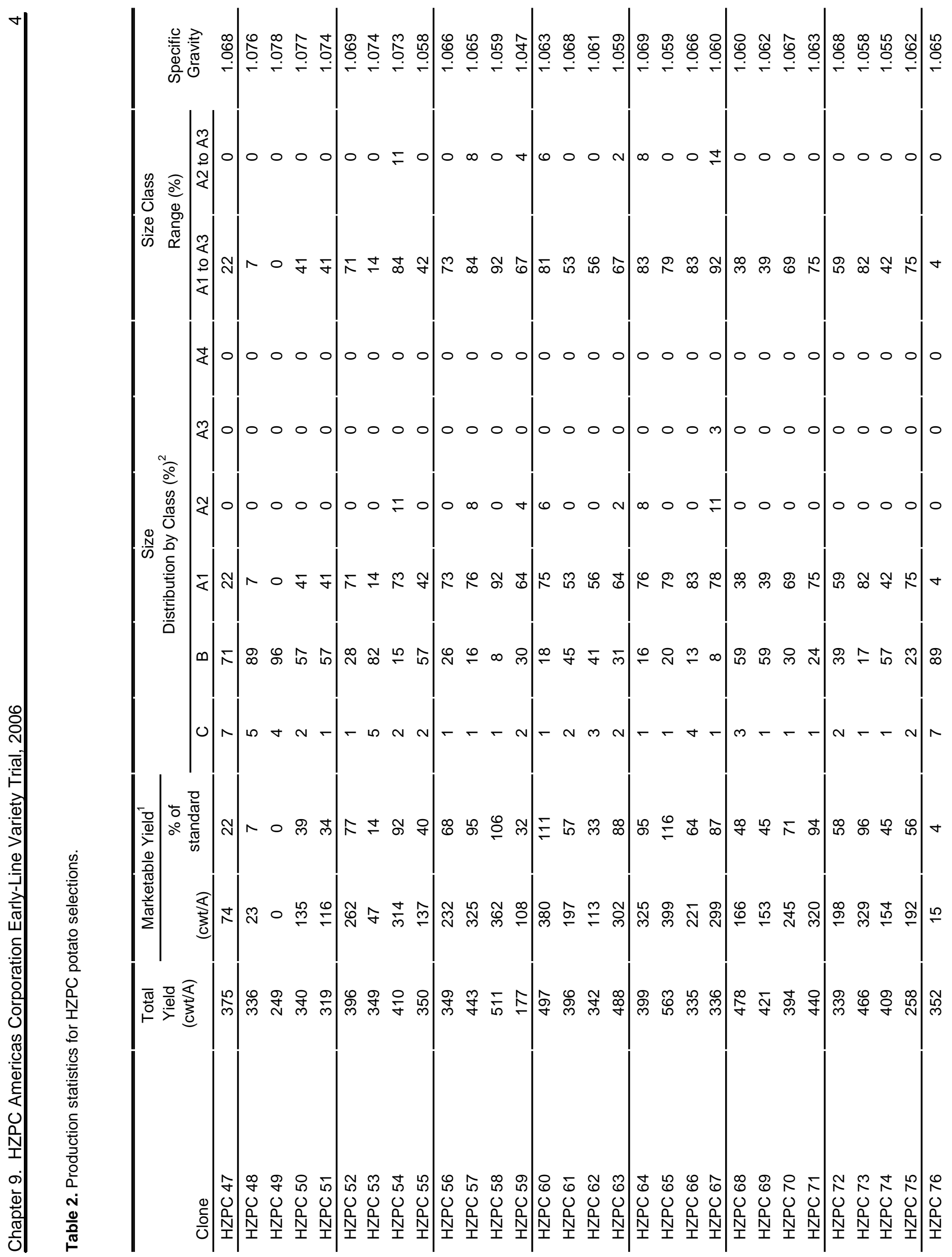




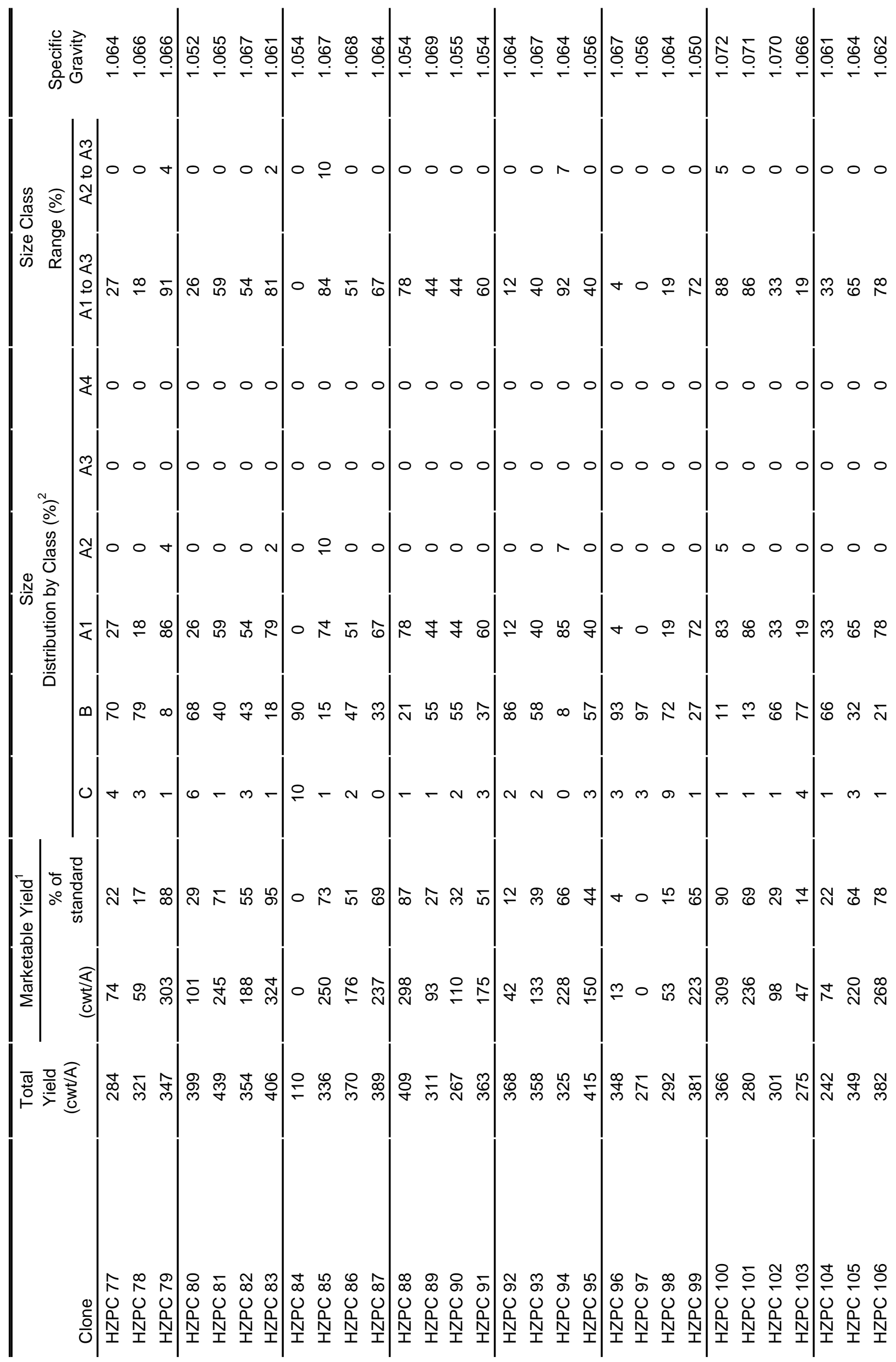




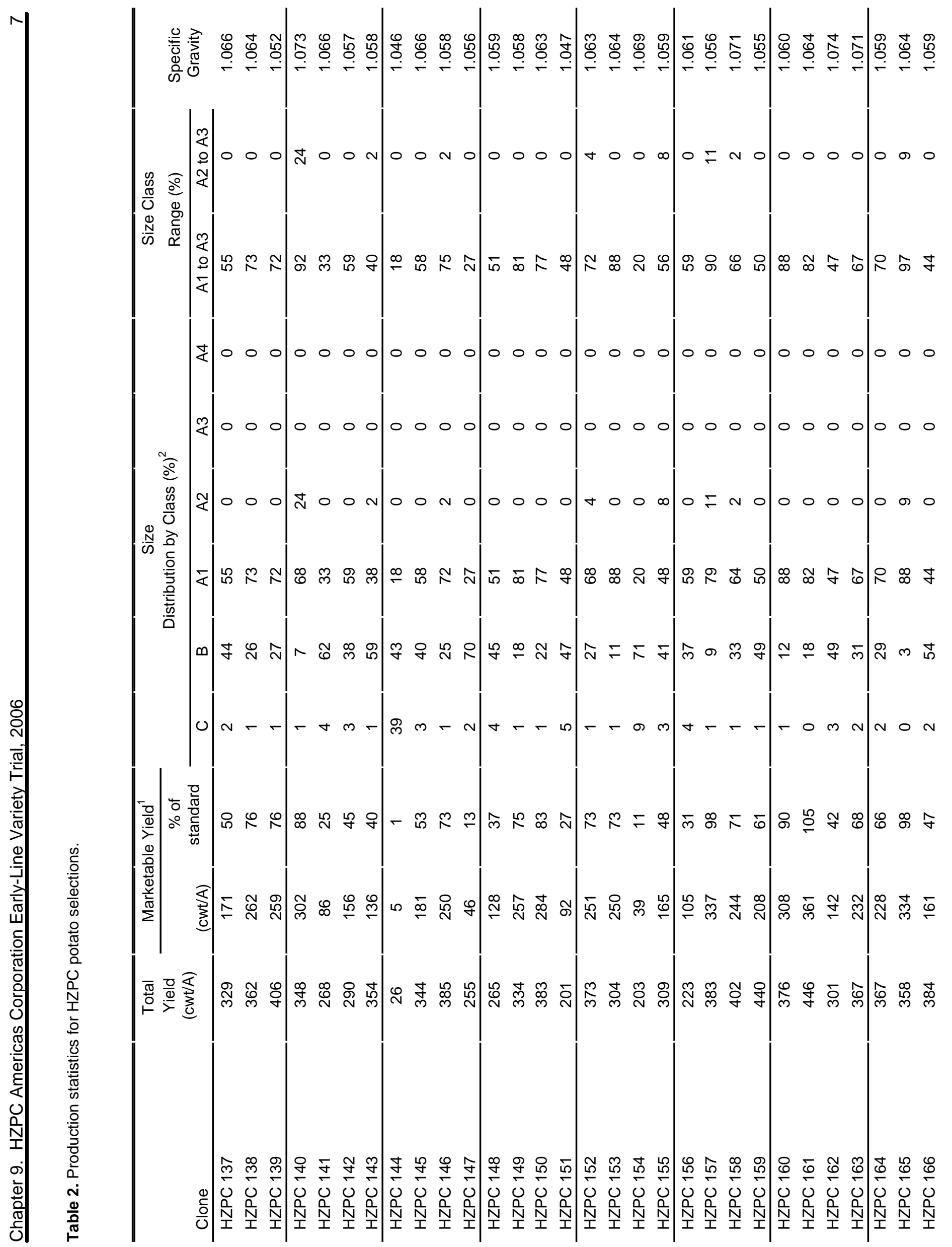




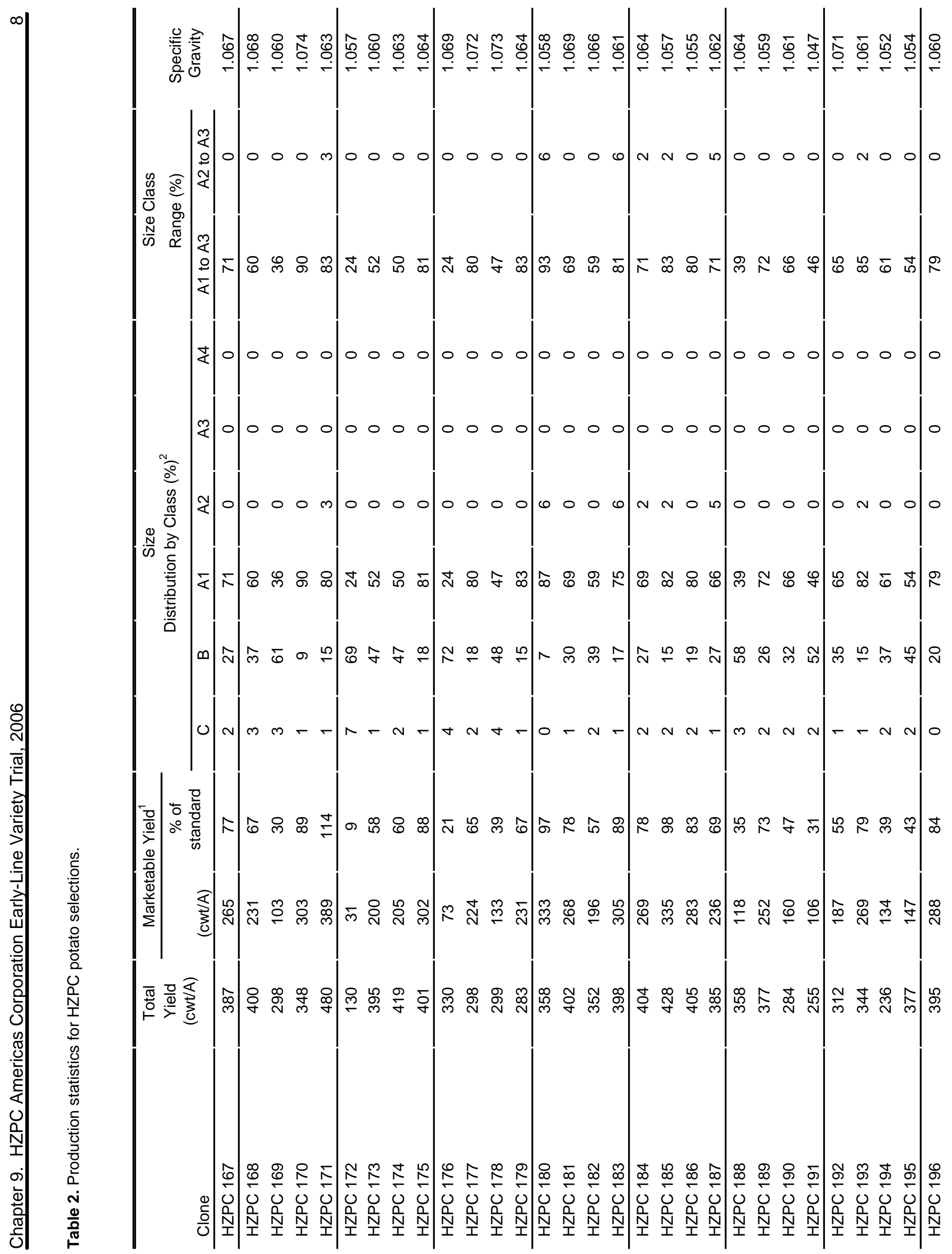




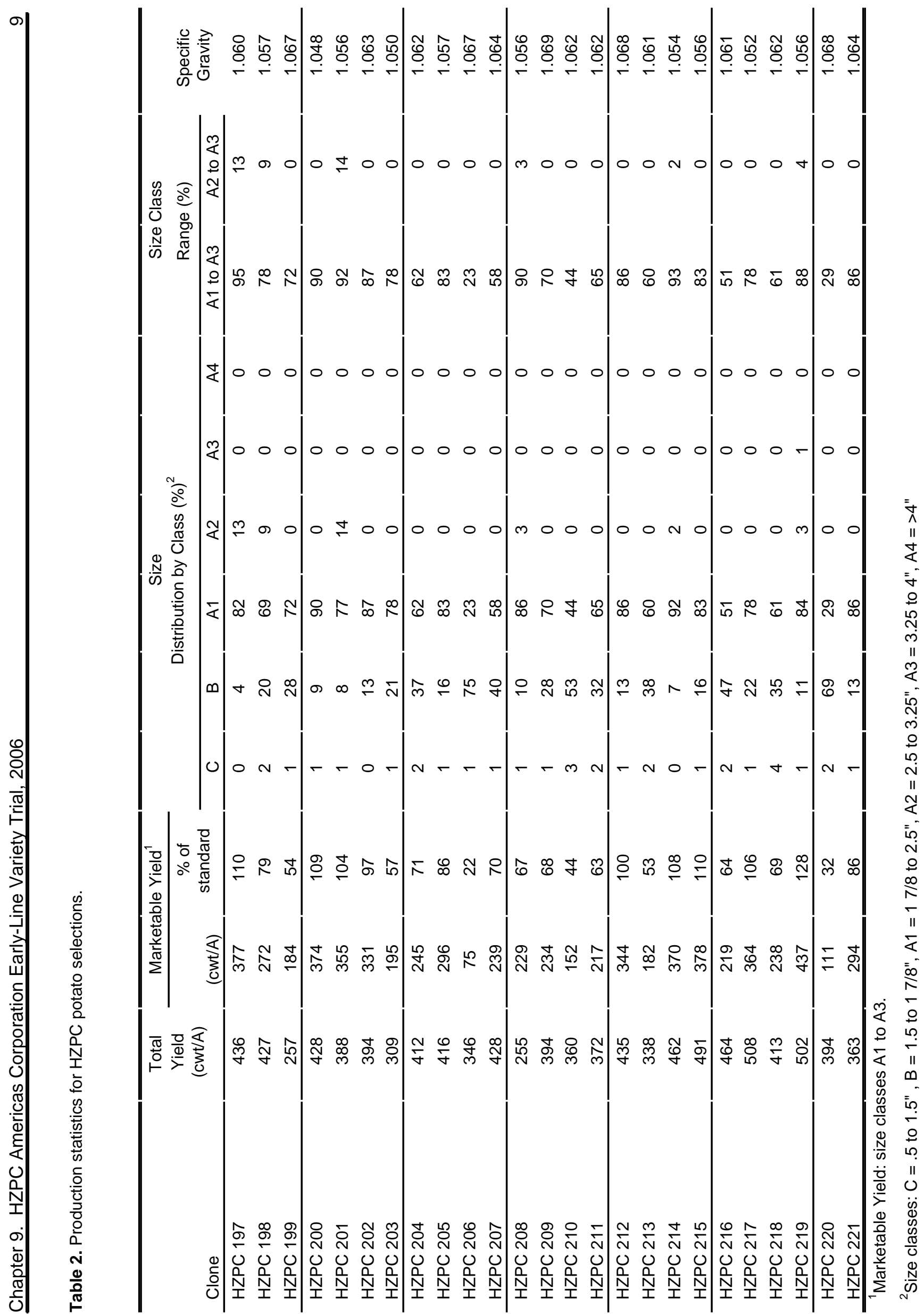


Table 3. Plant growth characteristics and tuber appearance of HZPC potato selections.

\begin{tabular}{|c|c|c|c|c|c|}
\hline \multirow[b]{2}{*}{ Clone } & \multicolumn{3}{|c|}{ Plant Growth Characteristics ${ }^{1}$} & \multirow[b]{2}{*}{$\begin{array}{l}\text { Vine } \\
\text { Maturity }\end{array}$} & \multirow[b]{2}{*}{$\begin{array}{l}\text { Tuber } \\
\text { Appearance }^{2}\end{array}$} \\
\hline & $\begin{array}{c}\% \\
\text { Stand }\end{array}$ & $\begin{array}{l}\text { Early } \\
\text { Vigor }\end{array}$ & $\begin{array}{l}\text { Vine } \\
\text { Type }\end{array}$ & & \\
\hline LaChipper & 100 & 7 & 9 & 7 & 6.0 \\
\hline Adora & 100 & 7 & 8 & 7 & 6.0 \\
\hline Atlantic & 100 & 6 & 8 & 9 & 6.0 \\
\hline Belrus & 100 & 5 & $8-9$ & 9 & 6.0 \\
\hline Fabula & 56 & 5 & $8-7$ & 9 & 5.5 \\
\hline Goldrush & 100 & 5 & 9 & 9 & 6.0 \\
\hline LaRouge & 100 & 6 & 9 & 7 & 6.0 \\
\hline Harley Blackwell & 94 & 6 & 9 & 8 & 5.5 \\
\hline Red LaSoda & 100 & 5 & 9 & 7 & 5.5 \\
\hline Red Pontiac & 100 & 6 & 9 & 7 & 4.5 \\
\hline Russet Burbank & 100 & 5 & 9 & 9 & 4.0 \\
\hline Sebago & 38 & 6 & 8 & 7 & 4.0 \\
\hline Yukon Gold & 63 & 6 & 9 & 7 & 5.0 \\
\hline HZPC 1 & 100 & 6 & 9 & 8 & 6.0 \\
\hline HZPC 2 & 100 & 8 & $9-6$ & 7 & 5.0 \\
\hline HZPC 3 & 100 & 6 & 8 & 6 & 5.5 \\
\hline HZPC 4 & 100 & 6 & 9 & 7 & 5.0 \\
\hline HZPC 5 & 100 & 8 & $8-5$ & 7 & 6.0 \\
\hline HZPC 6 & 100 & 6 & 5 & 6 & 7.0 \\
\hline HZPC 7 & 100 & 9 & 9 & 6 & 6.0 \\
\hline HZPC 8 & 100 & 9 & $9-6$ & 6 & 6.0 \\
\hline HZPC 9 & 94 & 9 & 6-9 & 5 & 6.5 \\
\hline HZPC 10 & 100 & 9 & $9-6$ & 6 & 6.0 \\
\hline HZPC 11 & 100 & 7 & 9 & 7 & 5.5 \\
\hline HZPC 12 & 100 & 8 & 9 & 5 & 5.0 \\
\hline HZPC 13 & 100 & 5 & 8 & 7 & 4.5 \\
\hline HZPC 14 & 100 & 6 & 8 & 7 & 6.0 \\
\hline HZPC 15 & 100 & 6 & 9 & 7 & 6.5 \\
\hline HZPC 16 & 100 & 6 & - & - & 3.5 \\
\hline HZPC 17 & 100 & 6 & - & - & 3.5 \\
\hline HZPC 18 & 100 & 9 & 9 & 6 & 5.0 \\
\hline HZPC 19 & 100 & 5 & 9 & 8 & 7.0 \\
\hline HZPC 20 & 100 & 6 & 8 & 8 & 5.0 \\
\hline HZPC 21 & 94 & 7 & 9 & 8 & 4.0 \\
\hline HZPC 22 & 100 & 5 & 9 & 7 & 5.5 \\
\hline HZPC 23 & 100 & 5 & $8-7$ & 6 & 5.5 \\
\hline HZPC 24 & 100 & 6 & 9 & 7 & 4.0 \\
\hline HZPC 25 & 100 & 6 & - & - & 3.0 \\
\hline HZPC 26 & 100 & 6 & na & 1 & na \\
\hline HZPC 27 & 94 & 6 & 9 & 7 & 5.0 \\
\hline HZPC 28 & 100 & 7 & 8-5 & 5 & 4.5 \\
\hline HZPC 29 & 100 & 7 & 9 & 7 & 5.5 \\
\hline HZPC 30 & 94 & 6 & 8 & 6 & 4.5 \\
\hline
\end{tabular}


Table 3. Plant growth characteristics and tuber appearance of HZPC potato selections.

\begin{tabular}{|c|c|c|c|c|c|}
\hline \multirow[b]{2}{*}{ Clone } & \multicolumn{3}{|c|}{ Plant Growth Characteristics ${ }^{1}$} & \multirow[b]{2}{*}{$\begin{array}{c}\text { Vine } \\
\text { Maturity }\end{array}$} & \multirow[b]{2}{*}{$\begin{array}{l}\text { Tuber } \\
\text { Appearance }^{2}\end{array}$} \\
\hline & $\begin{array}{c}\% \\
\text { Stand }\end{array}$ & $\begin{array}{l}\text { Early } \\
\text { Vigor }\end{array}$ & $\begin{array}{l}\text { Vine } \\
\text { Type }\end{array}$ & & \\
\hline HZPC 31 & 100 & 7 & 9 & 8 & 4.0 \\
\hline HZPC 32 & 100 & 5 & 8 & 7 & 4.0 \\
\hline HZPC 33 & 100 & 7 & 9 & 7 & 4.0 \\
\hline HZPC 34 & 100 & 8 & 9 & 7 & 4.0 \\
\hline HZPC 35 & 100 & 7 & 8 & 7 & 3.0 \\
\hline HZPC 36 & 100 & 5 & 9 & 7 & 4.0 \\
\hline HZPC 37 & 100 & 5 & - & - & 3.5 \\
\hline HZPC 38 & 94 & 6 & 9 & 7 & 5.0 \\
\hline HZPC 39 & 100 & 5 & 8 & 7 & 6.5 \\
\hline HZPC 40 & 94 & 7 & 9 & 7 & 7.0 \\
\hline HZPC 41 & 100 & 6 & 9 & 7 & 6.0 \\
\hline HZPC 42 & 100 & 5 & 8 & 8 & na \\
\hline HZPC 43 & 100 & 7 & $9-6$ & 8 & 4.5 \\
\hline HZPC 44 & 100 & 6 & 9 & 9 & 4.0 \\
\hline HZPC 45 & 100 & 5 & 9 & 8 & 6.5 \\
\hline HZPC 46 & 100 & 8 & 9 & 7 & 4.5 \\
\hline HZPC 47 & 100 & 7 & 9 & 7 & 6.0 \\
\hline HZPC 48 & 100 & 6 & 9 & 8 & 6.5 \\
\hline HZPC 49 & 100 & 7 & 9 & 7 & 7.0 \\
\hline HZPC 50 & 100 & 8 & 9 & 7 & 6.0 \\
\hline HZPC 51 & 100 & 7 & 9 & 6 & 5.5 \\
\hline HZPC 52 & 100 & 6 & 8 & 8 & 5.5 \\
\hline HZPC 53 & 100 & 7 & 9 & 8 & 7.0 \\
\hline HZPC 54 & 100 & 6 & 9 & 8 & 4.0 \\
\hline HZPC 55 & 100 & 6 & 8 & 6 & 4.5 \\
\hline HZPC 56 & 100 & 6 & 9 & 7 & 5.5 \\
\hline HZPC 57 & 100 & 7 & 9 & 8 & 6.0 \\
\hline HZPC 58 & 100 & 7 & 9 & 7 & 2.0 \\
\hline HZPC 59 & 100 & 6 & $8-7$ & 7 & 4.5 \\
\hline HZPC 60 & 100 & 7 & 9 & 8 & 6.5 \\
\hline HZPC 61 & 100 & 8 & 8 & 8 & 4.5 \\
\hline HZPC 62 & 100 & 6 & 9 & 7 & 5.0 \\
\hline HZPC 63 & 100 & 6 & 8 & 7 & 4.5 \\
\hline HZPC 64 & 94 & 6 & 9 & 7 & 4.5 \\
\hline HZPC 65 & 100 & 5 & 8 & 9 & 6.5 \\
\hline HZPC 66 & 100 & 5 & 9 & 8 & 4.5 \\
\hline HZPC 67 & 100 & 5 & $8-7$ & 9 & 5.5 \\
\hline HZPC 68 & 100 & 7 & 9 & 7 & 4.0 \\
\hline HZPC 69 & 100 & 6 & 9 & 7 & 5.0 \\
\hline HZPC 70 & 100 & 6 & 8 & 7 & 6.0 \\
\hline HZPC 71 & 100 & 7 & 9 & 7 & 7.0 \\
\hline HZPC 72 & 100 & 6 & 8 & 7 & 4.5 \\
\hline HZPC 73 & 100 & 7 & 9 & 7 & 6.0 \\
\hline HZPC 74 & 100 & 8 & 8 & 6 & 5.5 \\
\hline
\end{tabular}


Table 3. Plant growth characteristics and tuber appearance of HZPC potato selections.

\begin{tabular}{|c|c|c|c|c|c|}
\hline \multirow[b]{2}{*}{ Clone } & \multicolumn{3}{|c|}{ Plant Growth Characteristics ${ }^{1}$} & \multirow[b]{2}{*}{$\begin{array}{c}\text { Vine } \\
\text { Maturity }\end{array}$} & \multirow[b]{2}{*}{$\begin{array}{l}\text { Tuber } \\
\text { Appearance }\end{array}$} \\
\hline & $\begin{array}{c}\% \\
\text { Stand }\end{array}$ & $\begin{array}{l}\text { Early } \\
\text { Vigor }\end{array}$ & $\begin{array}{l}\text { Vine } \\
\text { Type }\end{array}$ & & \\
\hline HZPC 75 & 100 & 5 & 8 & 7 & 6.5 \\
\hline HZPC 76 & 94 & 8 & $8-5$ & 7 & 7.5 \\
\hline HZPC 77 & 100 & 7 & 9 & 7 & 7.0 \\
\hline HZPC 78 & 94 & 5 & 8 & 9 & 7.5 \\
\hline HZPC 79 & 100 & 5 & 9 & 7 & 6.5 \\
\hline HZPC 80 & 100 & 7 & $8-5$ & 8 & 7.5 \\
\hline HZPC 81 & 94 & 7 & $9-6$ & 6 & 6.0 \\
\hline HZPC 82 & 100 & 7 & $9-6$ & 6 & 6.0 \\
\hline HZPC 83 & 100 & 5 & 9 & 8 & 6.0 \\
\hline HZPC 84 & 100 & 6 & - & - & 5.5 \\
\hline HZPC 85 & 94 & 7 & 9 & 7 & 6.0 \\
\hline HZPC 86 & 94 & 7 & 8 & 7 & 5.5 \\
\hline HZPC 87 & 100 & 7 & 8 & 7 & 4.0 \\
\hline HZPC 88 & 100 & 6 & 9 & 7 & 4.0 \\
\hline HZPC 89 & 100 & 8 & $9-6$ & 6 & 4.5 \\
\hline HZPC 90 & 100 & 5 & 9 & 7 & 4.0 \\
\hline HZPC 91 & 100 & 7 & 9 & 6 & 4.0 \\
\hline HZPC 92 & 100 & 7 & 9 & 7 & 4.5 \\
\hline HZPC 93 & 100 & 7 & $9-6$ & 7 & 3.5 \\
\hline HZPC 94 & 94 & 9 & 8 & 7 & 4.0 \\
\hline HZPC 95 & 100 & 7 & 9 & 7 & 4.5 \\
\hline HZPC 96 & 94 & 6 & 9 & 8 & 5.0 \\
\hline HZPC 97 & 100 & 6 & 8 & 7 & 5.5 \\
\hline HZPC 98 & 100 & 5 & 8 & 7 & 6.0 \\
\hline HZPC 99 & 100 & 7 & 9 & 5 & 5.5 \\
\hline HZPC 100 & 100 & 7 & 8 & 7 & 5.5 \\
\hline HZPC 101 & 100 & 6 & 9 & 6 & 4.5 \\
\hline HZPC 102 & 100 & 6 & 8 & 7 & 4.5 \\
\hline HZPC 103 & 100 & 8 & 8 & 7 & 4.0 \\
\hline HZPC 104 & 100 & 6 & 8 & 7 & 4.0 \\
\hline HZPC 105 & 94 & 6 & 8 & 8 & 6.0 \\
\hline HZPC 106 & 100 & 6 & 9 & 6 & 5.5 \\
\hline HZPC 107 & 100 & 6 & - & - & 6.0 \\
\hline HZPC 108 & 94 & 7 & 8 & 7 & 6.0 \\
\hline HZPC 109 & 100 & 8 & 8 & 6 & 5.0 \\
\hline HZPC 110 & 100 & 8 & 9 & 7 & 6.5 \\
\hline HZPC 111 & 100 & 7 & 9 & 8 & 6.0 \\
\hline HZPC 112 & 100 & 8 & 9 & 8 & 6.5 \\
\hline HZPC 113 & 100 & 7 & 9 & 7 & 4.0 \\
\hline HZPC 114 & 100 & 8 & $9-6$ & 7 & 6.0 \\
\hline HZPC 115 & 100 & 9 & 9 & 7 & 6.5 \\
\hline HZPC 116 & 75 & 9 & $9-6$ & 7 & 4.5 \\
\hline HZPC 117 & 88 & 6 & 9 & 7 & 4.5 \\
\hline HZPC 118 & 100 & 8 & 9 & 7 & 4.5 \\
\hline
\end{tabular}


Table 3. Plant growth characteristics and tuber appearance of HZPC potato selections.

\begin{tabular}{|c|c|c|c|c|c|}
\hline \multirow[b]{2}{*}{ Clone } & \multicolumn{3}{|c|}{ Plant Growth Characteristics ${ }^{1}$} & \multirow[b]{2}{*}{$\begin{array}{c}\text { Vine } \\
\text { Maturity }\end{array}$} & \multirow[b]{2}{*}{$\begin{array}{l}\text { Tuber } \\
\text { Appearance }^{2}\end{array}$} \\
\hline & $\begin{array}{c}\% \\
\text { Stand }\end{array}$ & $\begin{array}{l}\text { Early } \\
\text { Vigor }\end{array}$ & $\begin{array}{l}\text { Vine } \\
\text { Type }\end{array}$ & & \\
\hline HZPC 119 & 94 & 6 & 9 & 7 & 5.0 \\
\hline HZPC 120 & 100 & 7 & 9 & 8 & 5.0 \\
\hline HZPC 121 & 100 & 7 & 9 & 7 & 3.0 \\
\hline HZPC 122 & 94 & 7 & 8 & 7 & 4.0 \\
\hline HZPC 123 & 100 & 7 & 8 & 6 & 3.0 \\
\hline HZPC 124 & 100 & 5 & - & - & 3.0 \\
\hline HZPC 125 & 94 & 5 & - & - & 3.5 \\
\hline HZPC 126 & 100 & 4 & 9 & 7 & 3.5 \\
\hline HZPC 127 & 100 & 5 & 8 & 7 & 6.5 \\
\hline HZPC 128 & 94 & 5 & 9 & 7 & 5.5 \\
\hline HZPC 129 & 94 & 7 & 9 & 9 & 5.5 \\
\hline HZPC 130 & 100 & 6 & 8 & 6 & 6.0 \\
\hline HZPC 131 & 81 & 8 & 9 & 7 & 3.5 \\
\hline HZPC 132 & 94 & 6 & 9 & 7 & 3.5 \\
\hline HZPC 133 & 94 & 5 & 9 & 7 & 3.5 \\
\hline HZPC 134 & 88 & 7 & 9 & 7 & 4.5 \\
\hline HZPC 135 & 88 & 5 & 8 & 7 & 4.0 \\
\hline HZPC 136 & 94 & 6 & 8 & 9 & 4.5 \\
\hline HZPC 137 & 88 & 5 & 7 & 9 & 5.0 \\
\hline HZPC 138 & 100 & 5 & 8 & 8 & 5.5 \\
\hline HZPC 139 & 94 & 6 & 8 & 8 & 5.0 \\
\hline HZPC 140 & 100 & 5 & 8 & 7 & 5.5 \\
\hline HZPC 141 & 94 & 6 & 9 & 6 & 4.0 \\
\hline HZPC 142 & 100 & 7 & 8 & 7 & 4.0 \\
\hline HZPC 143 & 100 & 5 & 9 & 7 & 4.0 \\
\hline HZPC 144 & 100 & 5 & - & - & 2.0 \\
\hline HZPC 145 & 100 & 6 & 8 & 7 & 4.0 \\
\hline HZPC 146 & 100 & 6 & 8 & 7 & 4.0 \\
\hline HZPC 147 & 56 & 6 & 8 & 8 & 3.5 \\
\hline HZPC 148 & 100 & 6 & 8 & 7 & 4.0 \\
\hline HZPC 149 & 100 & 7 & 8 & 6 & 6.5 \\
\hline HZPC 150 & 100 & 6 & 9 & 8 & 6.0 \\
\hline HZPC 151 & 94 & 5 & 8 & 8 & 4.5 \\
\hline HZPC 152 & 100 & 6 & 8 & 9 & 4.5 \\
\hline HZPC 153 & 88 & 6 & 8 & 7 & 5.5 \\
\hline HZPC 154 & 100 & 7 & 8 & 7 & 4.5 \\
\hline HZPC 155 & 88 & 5 & 8 & 7 & 4.0 \\
\hline HZPC 156 & 56 & 4 & 7 & 7 & 4.0 \\
\hline HZPC 157 & 94 & 6 & 8 & 9 & 3.5 \\
\hline HZPC 158 & 100 & 6 & 9 & 7 & 4.0 \\
\hline HZPC 159 & 94 & 6 & 9 & 7 & 4.0 \\
\hline HZPC 160 & 100 & 6 & 8 & 6 & 4.5 \\
\hline HZPC 161 & 100 & 6 & 9 & 7 & 4.5 \\
\hline HZPC 162 & 100 & 6 & 8 & 7 & 4.5 \\
\hline
\end{tabular}


Table 3. Plant growth characteristics and tuber appearance of HZPC potato selections.

\begin{tabular}{|c|c|c|c|c|c|}
\hline \multirow[b]{2}{*}{ Clone } & \multicolumn{3}{|c|}{ Plant Growth Characteristics ${ }^{1}$} & \multirow[b]{2}{*}{$\begin{array}{c}\text { Vine } \\
\text { Maturity }\end{array}$} & \multirow[b]{2}{*}{$\begin{array}{l}\text { Tuber } \\
\text { Appearance }\end{array}$} \\
\hline & $\begin{array}{c}\% \\
\text { Stand }\end{array}$ & $\begin{array}{l}\text { Early } \\
\text { Vigor }\end{array}$ & $\begin{array}{l}\text { Vine } \\
\text { Type }\end{array}$ & & \\
\hline HZPC 163 & 100 & 7 & 6 & 6 & 5.0 \\
\hline HZPC 164 & 100 & 6 & 9 & 7 & 4.5 \\
\hline HZPC 165 & 100 & 7 & 9 & 6 & 6.0 \\
\hline HZPC 166 & 100 & 5 & 8 & 7 & 5.5 \\
\hline HZPC 167 & 100 & 6 & 9 & 7 & 5.5 \\
\hline HZPC 168 & 100 & 6 & 8 & 7 & 6.0 \\
\hline HZPC 169 & 100 & 5 & 8 & 6 & 6.0 \\
\hline HZPC 170 & 100 & 7 & 9 & 7 & 6.0 \\
\hline HZPC 171 & 100 & 6 & 9 & 8 & 5.5 \\
\hline HZPC 172 & 100 & 6 & 8 & 6 & 4.0 \\
\hline HZPC 173 & 100 & 6 & 9 & 7 & 3.5 \\
\hline HZPC 174 & 94 & 7 & 9 & 7 & 4.0 \\
\hline HZPC 175 & 100 & 7 & 9 & 7 & 4.0 \\
\hline HZPC 176 & 100 & 5 & 9 & 7 & 4.0 \\
\hline HZPC 177 & 100 & 4 & 8 & 8 & 4.5 \\
\hline HZPC 178 & 94 & 5 & 7 & 9 & 6.5 \\
\hline HZPC 179 & 100 & 3 & 8 & 9 & 5.0 \\
\hline HZPC 180 & 100 & 5 & 9 & 7 & 4.5 \\
\hline HZPC 181 & 100 & 6 & 9 & 9 & 5.0 \\
\hline HZPC 182 & 100 & 5 & 8 & 9 & 3.5 \\
\hline HZPC 183 & 100 & 5 & 8 & 9 & 5.0 \\
\hline HZPC 184 & 100 & 7 & $8-9$ & 7 & 5.5 \\
\hline HZPC 185 & 100 & 6 & 8 & 7 & 4.5 \\
\hline HZPC 186 & 88 & 6 & 8 & 9 & 4.0 \\
\hline HZPC 187 & 100 & 5 & 8 & 9 & 6.0 \\
\hline HZPC 188 & 94 & 5 & 9 & 6 & 6.0 \\
\hline HZPC 189 & 100 & 7 & 9 & 7 & 5.5 \\
\hline HZPC 190 & 81 & 5 & 8 & 7 & 4.5 \\
\hline HZPC 191 & 88 & 7 & 9 & 5 & 4.5 \\
\hline HZPC 192 & 100 & 5 & 8 & 8 & 4.5 \\
\hline HZPC 193 & 94 & 6 & 8 & 8 & 4.5 \\
\hline HZPC 194 & 94 & 5 & 7 & 6 & 6.0 \\
\hline HZPC 195 & 94 & 4 & 8 & 7 & 5.5 \\
\hline HZPC 196 & 100 & 7 & 9 & 7 & 5.5 \\
\hline HZPC 197 & 100 & 5 & 8 & 8 & 5.0 \\
\hline HZPC 198 & 100 & 6 & 9 & 8 & 4.5 \\
\hline HZPC 199 & 100 & 5 & 9 & 6 & 4.5 \\
\hline HZPC 200 & 100 & 4 & 8 & 9 & 4.0 \\
\hline HZPC 201 & 100 & 5 & 8 & 7 & 6.0 \\
\hline HZPC 202 & 100 & 7 & 8 & 7 & 5.0 \\
\hline HZPC 203 & 100 & 6 & 8 & 7 & 5.0 \\
\hline HZPC 204 & 100 & 6 & 8 & 8 & 5.5 \\
\hline HZPC 205 & 94 & 5 & 8 & 7 & 5.5 \\
\hline HZPC 206 & 75 & 6 & 9 & 9 & 6.5 \\
\hline
\end{tabular}


Table 3. Plant growth characteristics and tuber appearance of HZPC potato selections.

\begin{tabular}{lccccc} 
& \multicolumn{2}{c}{ Plant Growth Characteristics $^{1}$} & & \\
\cline { 2 - 5 } Clone & $\%$ & Early & Vine & Vine & Tuber \\
Stand & Vigor & Type & Maturity & Appearance $^{2}$ \\
\hline HZPC 207 & 100 & 7 & 9 & 8 & 6.0 \\
\hline HZPC 208 & 100 & 4 & 8 & 9 & 6.0 \\
HZPC 209 & 100 & 6 & 8 & 7 & 5.5 \\
HZPC 210 & 94 & 5 & 8 & 8 & 5.0 \\
HZPC 211 & 100 & 5 & 9 & 9 & 4.5 \\
\hline HZPC 212 & 100 & 7 & $9-6$ & 8 & 5.5 \\
HZPC 213 & 100 & 5 & 8 & 8 & 5.0 \\
HZPC 214 & 100 & 5 & 8 & 8 & 4.0 \\
HZPC 215 & 100 & 5 & 8 & 8 & 2.5 \\
\hline HZPC 216 & 100 & 5 & 9 & 8 & 6.0 \\
HZPC 217 & 100 & 6 & 8 & 8 & 4.5 \\
HZPC 218 & 94 & 6 & 9 & 9 & 5.0 \\
HZPC 219 & 94 & 5 & 9 & 9 & 6.0 \\
\hline HZPC 220 & 100 & 6 & 9 & 9 & 5.5 \\
HZPC 221 & 100 & 5 & 8 & 9 & 6.0 \\
\hline
\end{tabular}

${ }^{1}$ See rating system outlined in Table 1 (page 8).

${ }^{2}$ See rating system outlined in Table 2 (page 9). 


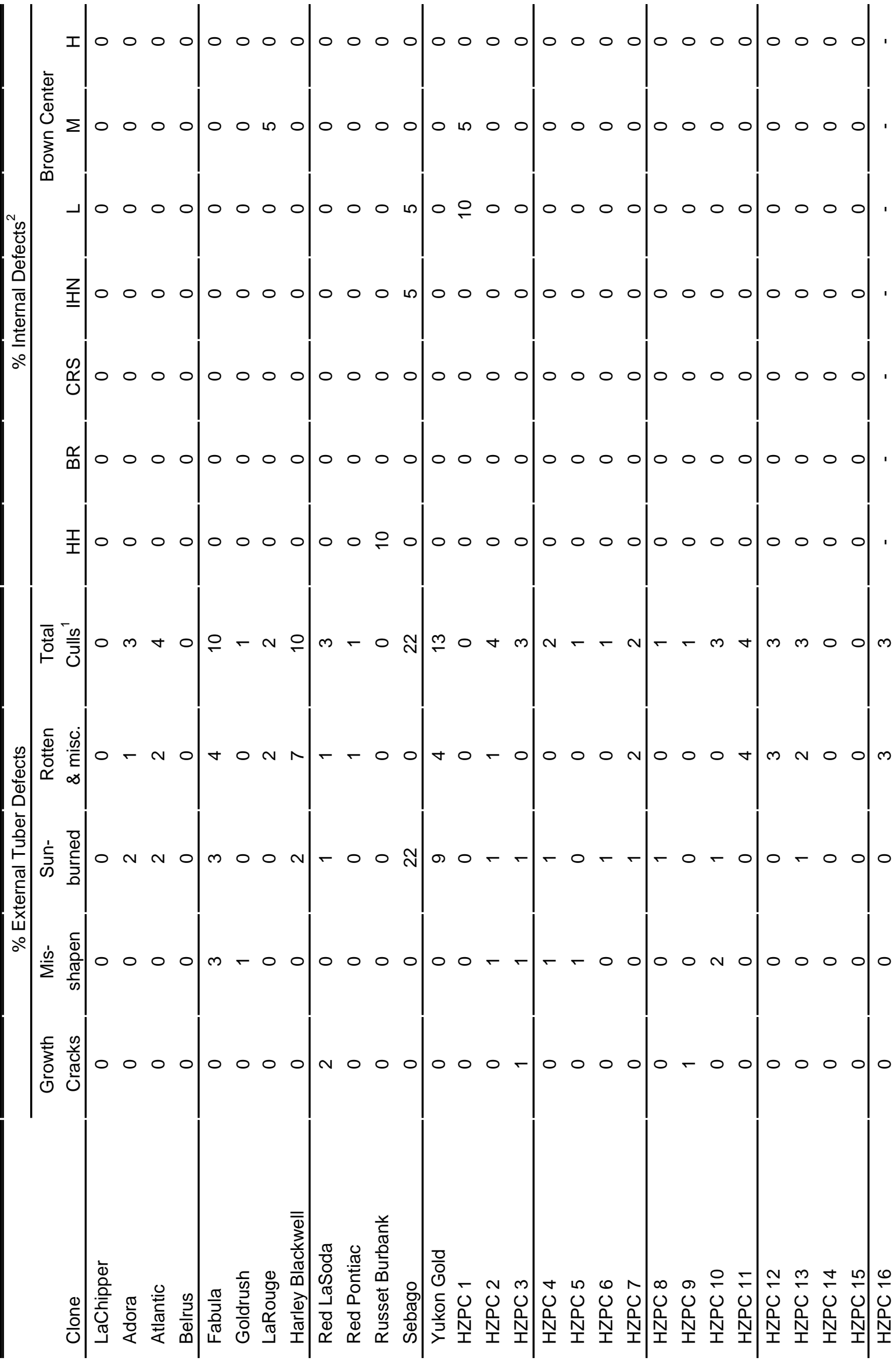




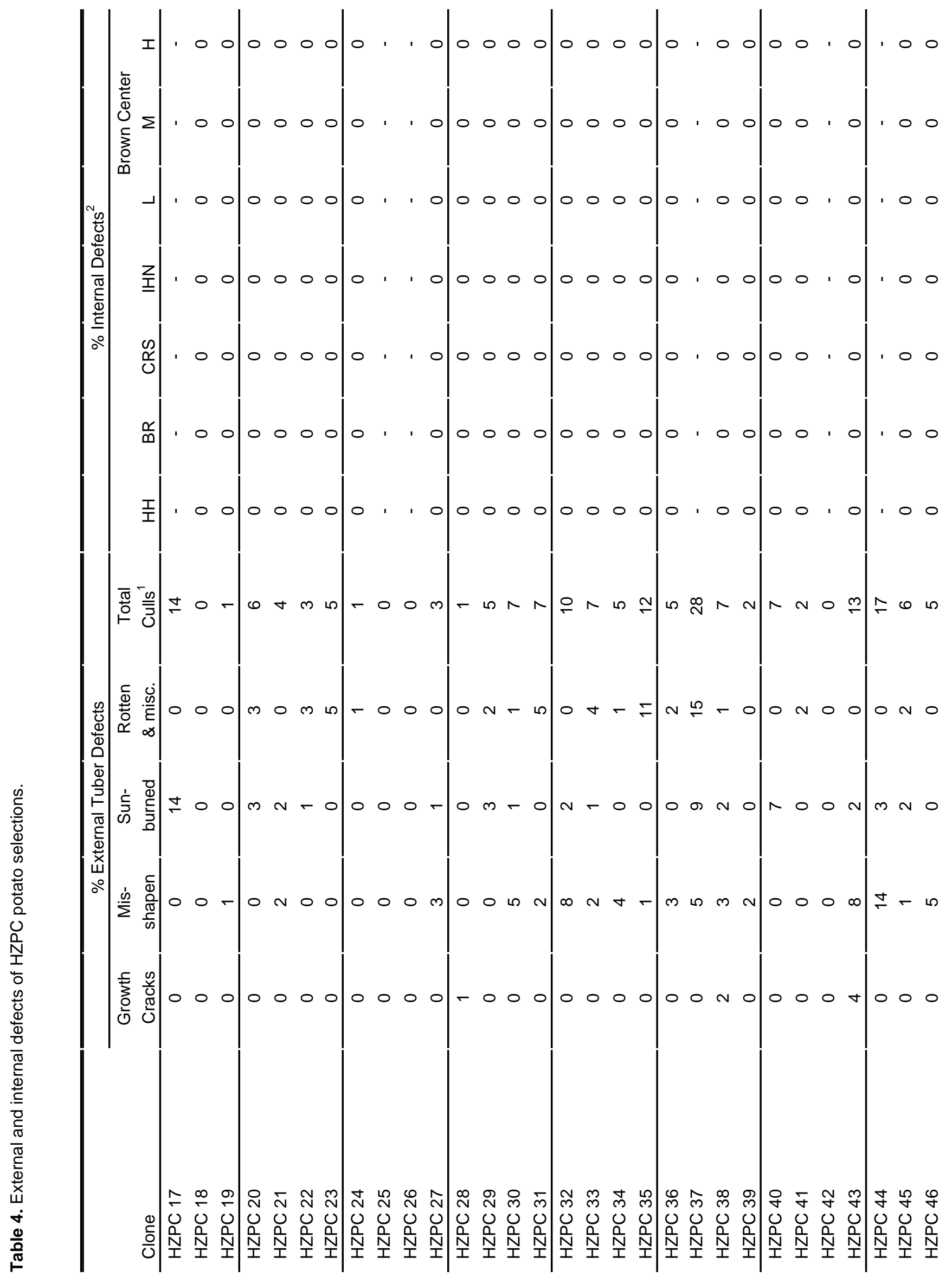




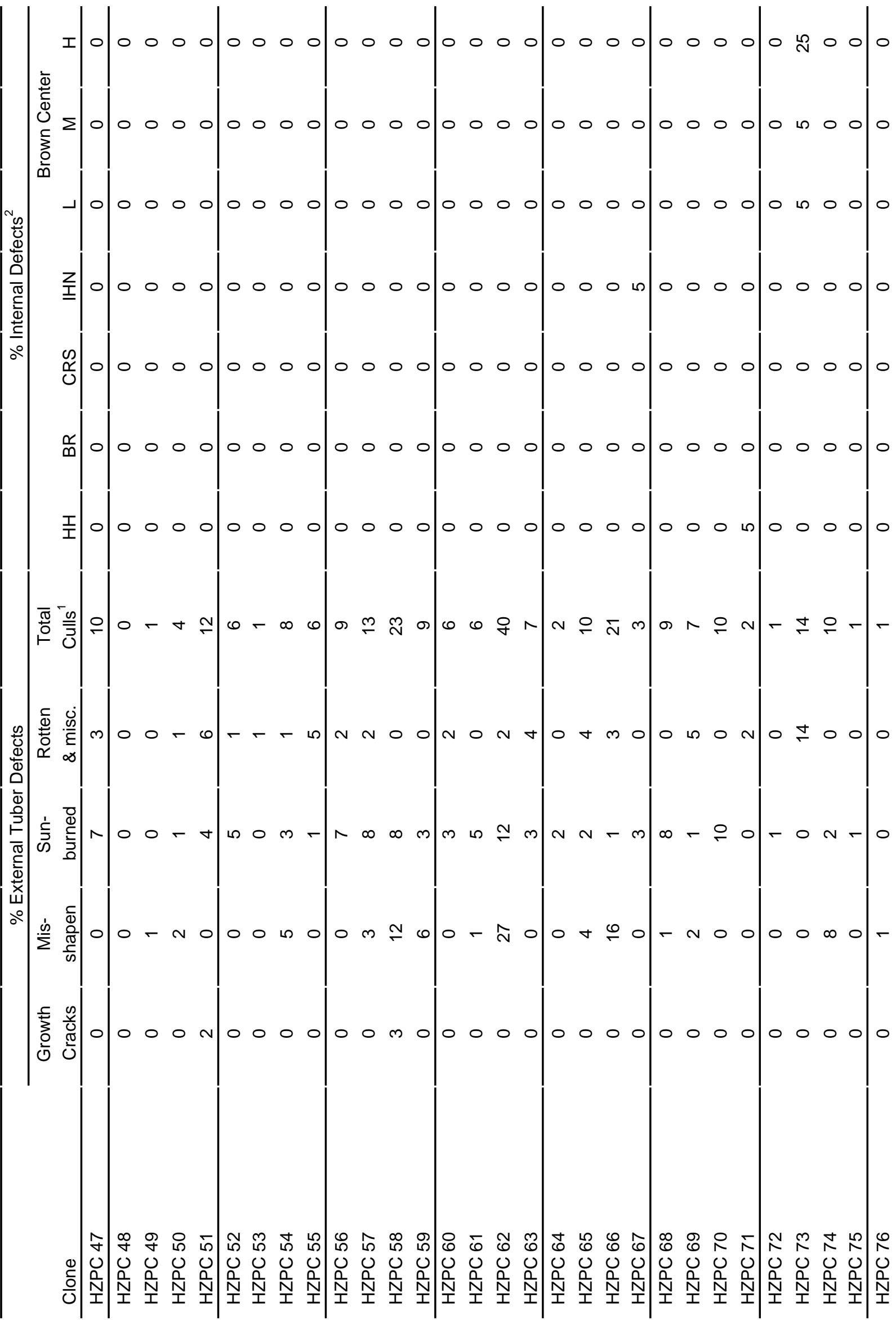




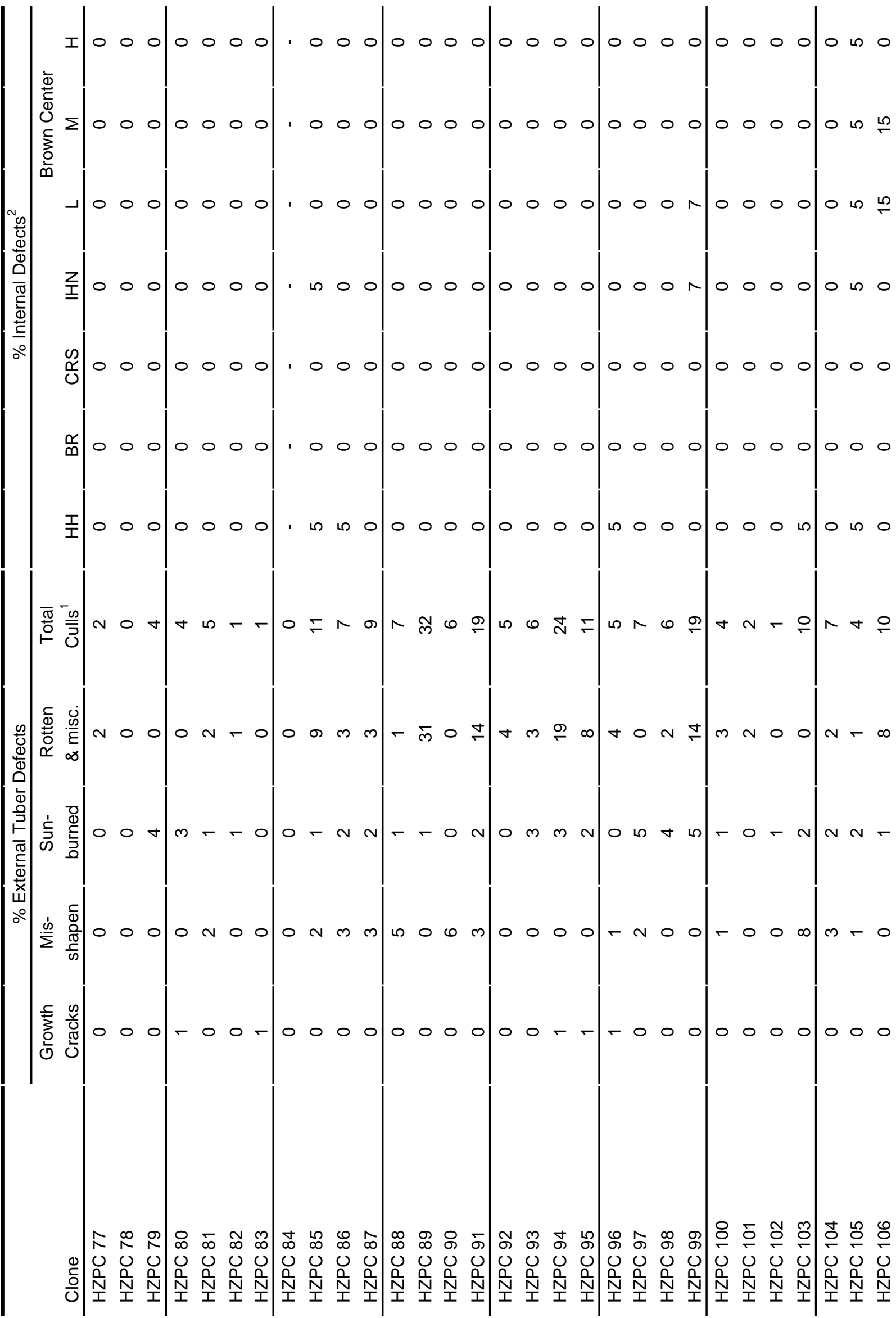




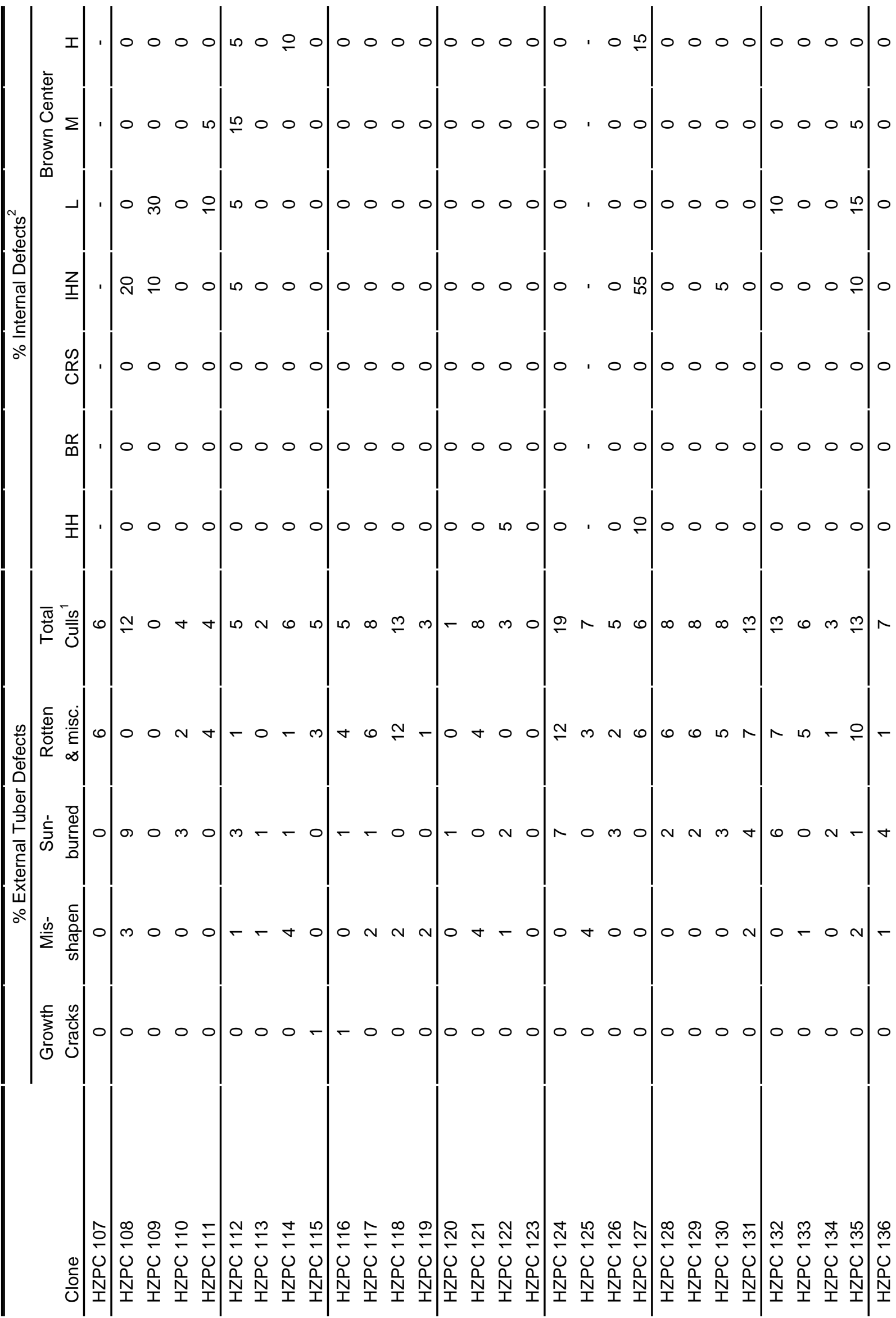




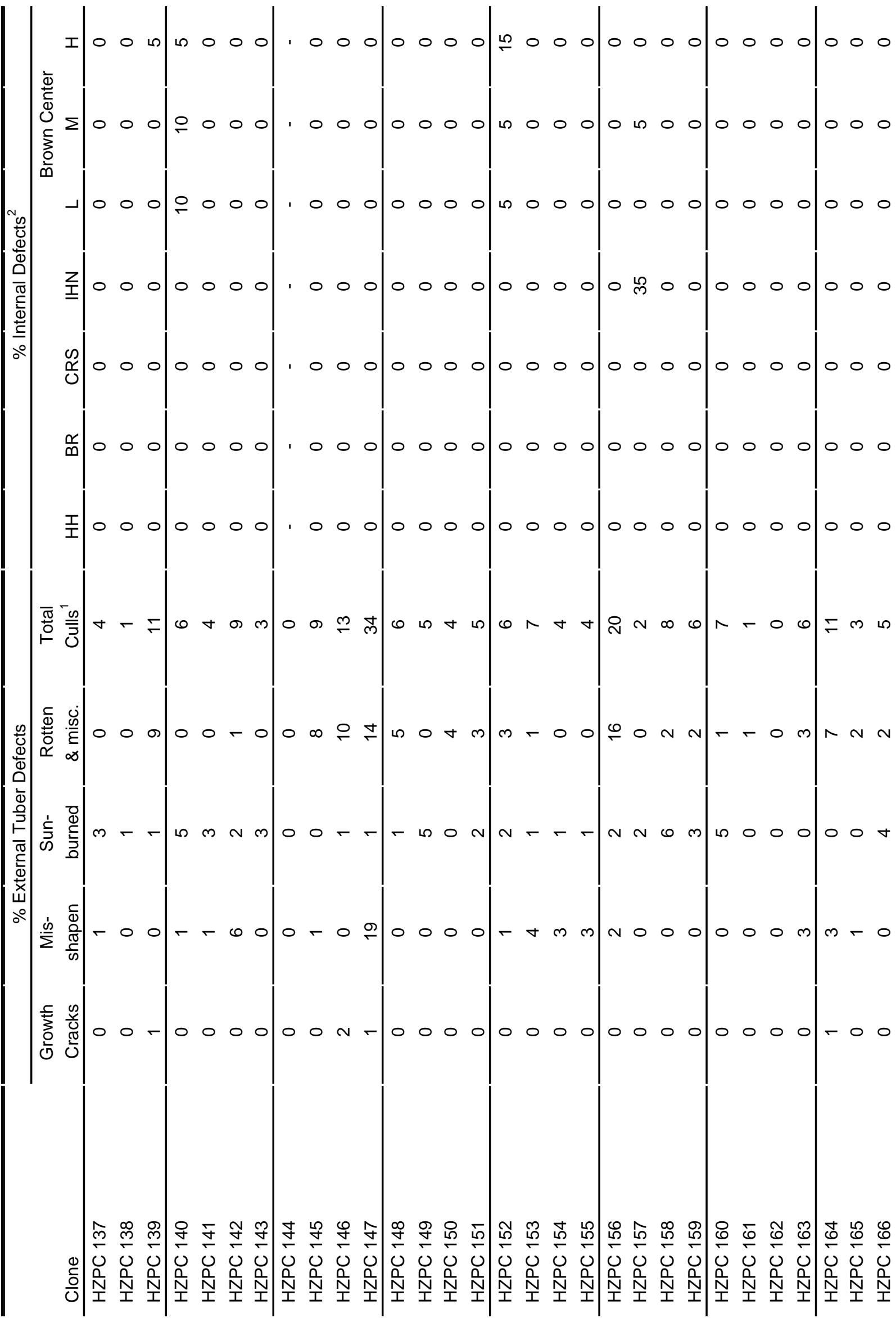




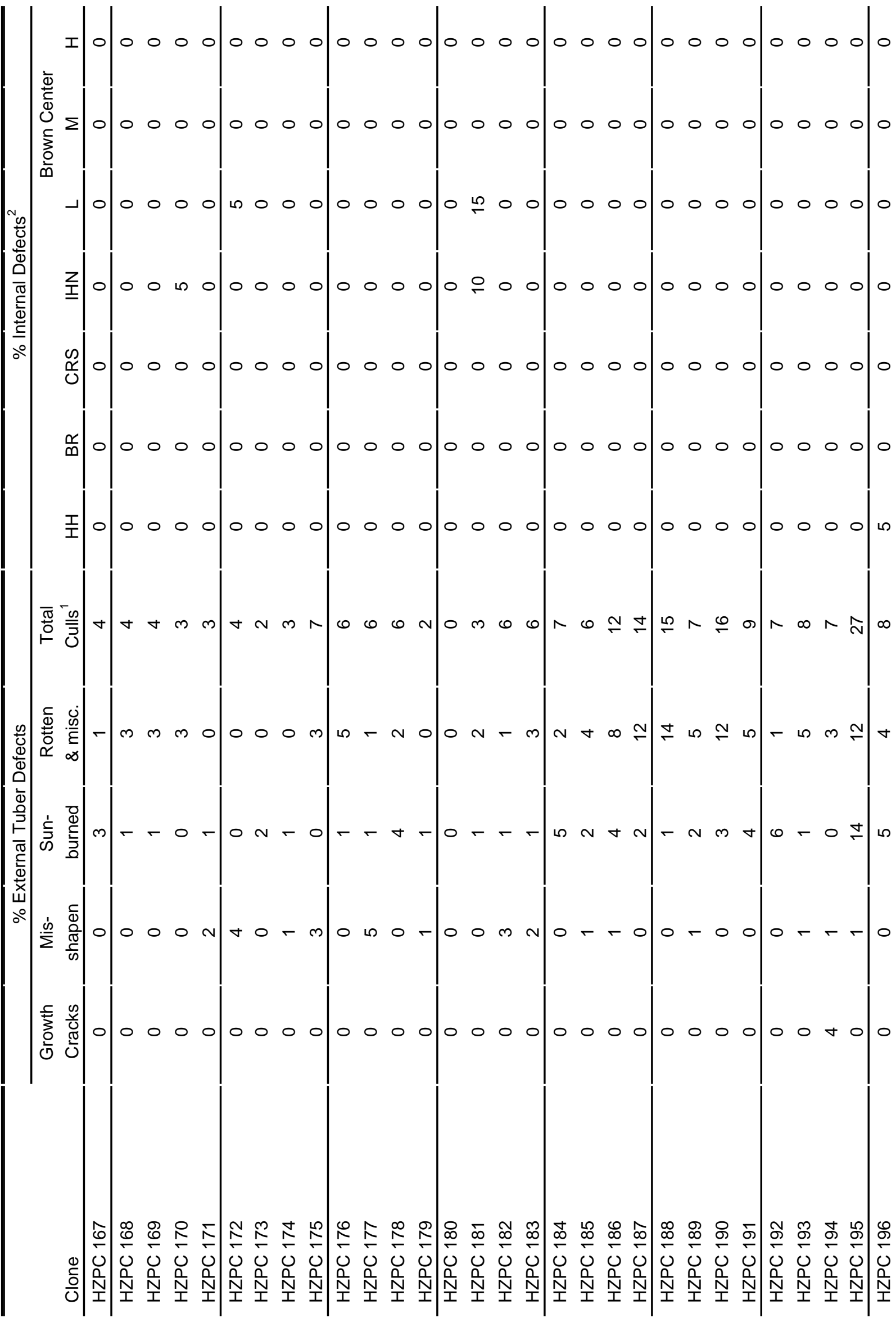




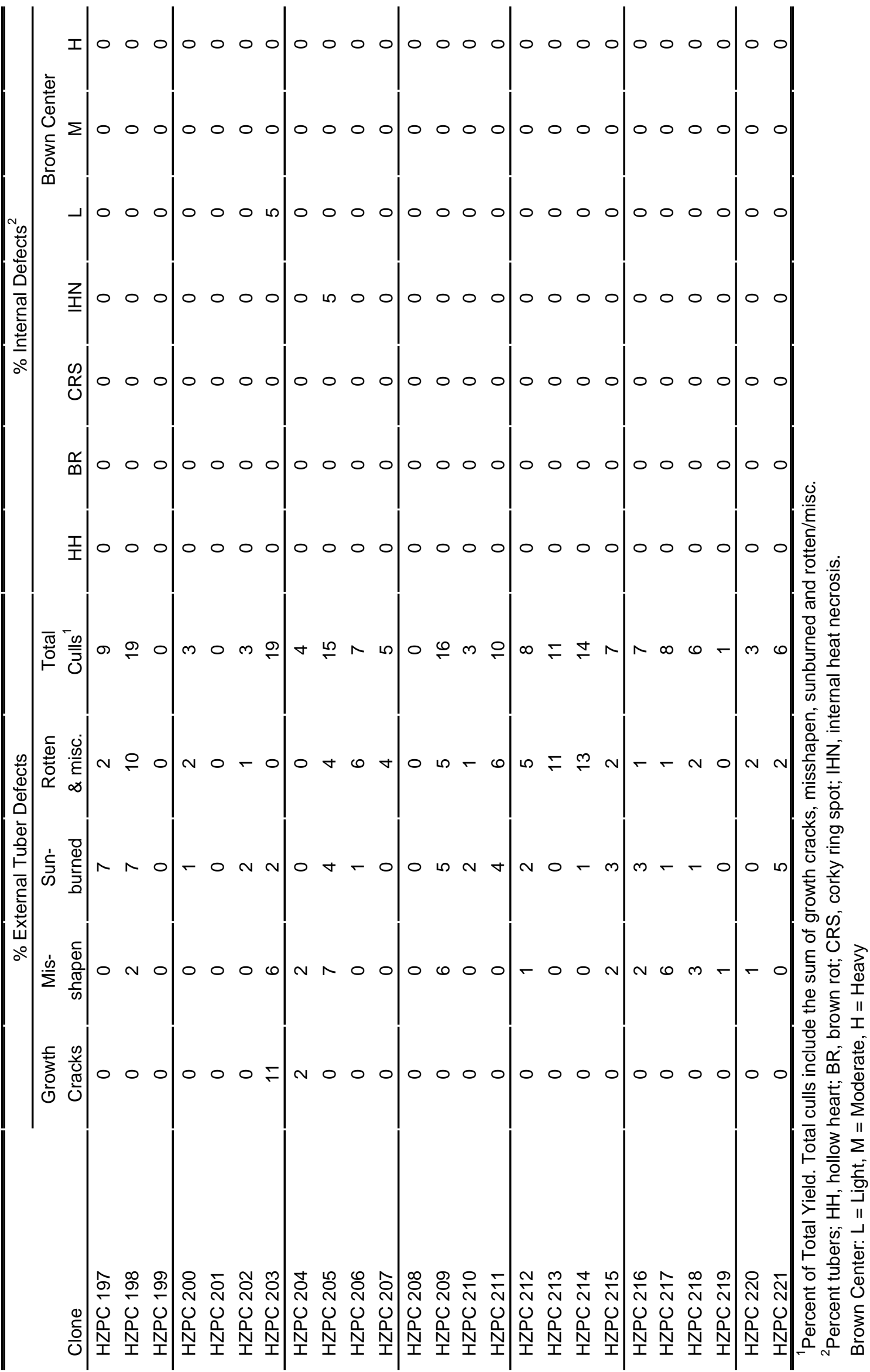

